\title{
How Artificial Intelligence Can Improve Understanding in Challenging Chaotic Environments
}

\author{
Reza Hafezi ${ }^{\text {a* }}$ \\ ${ }^{a}$ Futures Studies Research Group, National Research Institute for Science Policy (NRISP), Tehran, Iran. \\ *Corresponding Authors email: Hafezi (r.hafezi@aut.ac.ir)
}

\begin{abstract}
:
Decision-makers are concerned with the inherent complexity of the modern world's markets. However, price fluctuations, environmental concerns, technological development, emerging markets, political challenges, and social expectations made the $21^{\text {st }}$ century's more dynamic and complex. From a policy-making perspective, it is vital to uncover future trends. This paper proposed that artificial intelligence can improve interpretations in complex markets, such as financial and energy markets. In a complex environment, it is critical to investigate maximum available input features to ensure no valuable informative feature is neglected. Some AI-based models are investigated and presented that AI-based models can successfully uncover future trends. From a scenario development perspective purified input features subset refer to driving forces which shape alternative futures. Results showed that using AI can improve our understanding of how input features influence future behaviors and simultaneously improves prediction accuracy and reliability.
\end{abstract}

Keywords: prediction; futures studies; complex environment; machine learning data mining

\section{Introduction:}

Futures studies attracted attention both in the form of practice and theory. Although planning for a favorable future is an inherent characteristic of mankind, however, the modern world has brought some challenges. $21^{\text {st }}$ century's complexity rose based on numerous interconnected actors/agents with rarely predictable and some times with conflict of interests. Since resources are limited and competition has increased (in national, organizational and even individual levels) futures studies (FS) emerged as a scientific field aimed to deal with uncertainties in a complex and chaotic environment (Hafezi, Malekifar, and Akhavan 2018). As Hafezi et al. noted FS generally divided into two categories, prediction, and foresight (Hafezi, Malekifar, and Akhavan 2018). However, a more detailed view proposes three categories contain forecasting, modeling, and foresight. Forecasting and modeling deal with exploring future trends based on the existing history while foresight is an action-oriented and participatory process likewise other two emphasizes on open futures. Foresight studies have been intensively discussed in the literature in many aspects and forms in the last years (Meissner 2012). The most frequently applied future-oriented approaches are foresight, technology forecasting and technology assessment (Meissner 2012, Zweck 2002). Implementing foresight programs is meaningful at any stage: personal, organizational, local, subnational, national and international 
levels. Over the last decades, many foresight attempts have been made at the national level (Martin and Johnston 1999, Vecchiato and Roveda 2014, Meissner 2012, Prior et al. 2013).

A foresight activity does not aim at predicting a single pre-determined future (based on historical data) but it contains a participatory process to involve players and determine decisions which are taken today to shape various possible futures. Foresight offers possibilities to identify and take advantage of opportunities; to investigate and understand the nature of risks which are inherent in the innovation process and to develop reactions to mitigate the problems once they start to unfold (de Miranda Santo et al. 2006). Futures studies are known as an interdisciplinary field but yet its most well-used methodologies borrowed from management studies and as a result composed of qualitative methodologies. FS aimed to address problems when uncertainty rose and the environment transformed into a vague complex one. In such a situation it is not easy to cope with changes as predictability level reduced and thus the system is facing a higher risk. FS has a series of solutions. For instance, Popper proposed a systematic way to design a foresight program which only indicated only a few quantitative methods contain modeling, extrapolation, benchmarking and etc. (Popper 2008b, a). Also Smith and Saritas (Smith and Saritas 2011), Vishnevskiy and Egorova (Vishnevskiy and Egorova 2015), Miles (Ian Miles 2002), Keenan and Popper (Keenan and Popper 2007) proposed procedures to develop methodologies in order to study alternative futures, however mostly pure qualitative methods had formed methodologies. There is a logical answer which formerly has been mentioned by some researchers. As noted by Amer et al. qualitative methods are more suitable to project long run futures however quantitative methods are not reliable and may fail in longrun forecasts, while they are more reliable when used to project near futures (see figure 1) (Amer, Daim, and Jetter 2013). Both qualitative and quantitative approaches contain major weaknesses. The main weakness of qualitative approaches is that they are overly dependent on experts and their judgments. Consequently, it is important to organize efficient multidisciplinary expert panels to achieve output validity and quality and avoid bias and subjectivity. However, qualitative approaches are typically more flexible and more innovative, enabling them to focus on longer-term outlooks in comparison with quantitative approaches (Pillkahn 2008).

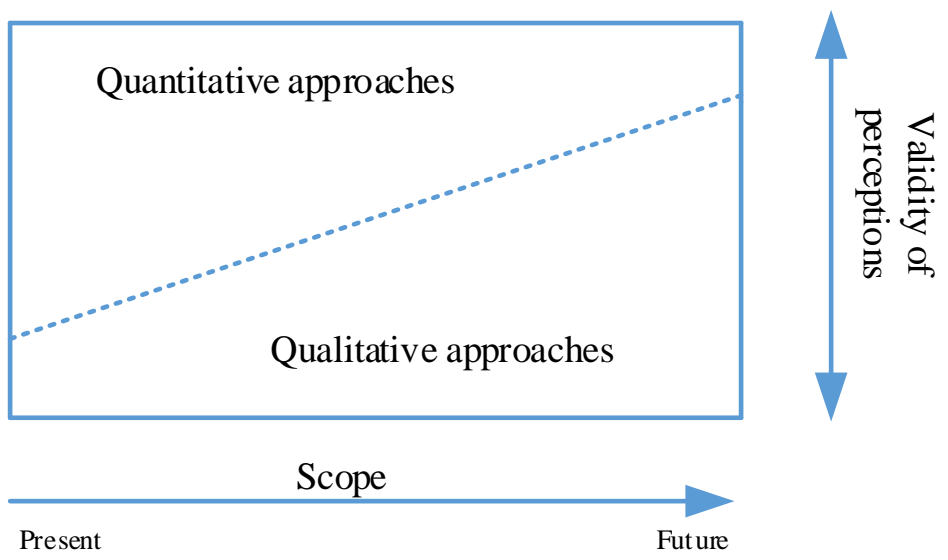

Figure 1. Validity of qualitative versus quantitative scenario approaches over time (adapted from (Amer, Daim, and Jetter 2013)) 
The main weaknesses of quantitative approaches are that they tend to be biased to the existence of valid, historical data and their trends. They also tend to be constrained to the information extracted from such data. That often unduly confines the problem/research space. As a consequence, some of the possible disruptive signals located in emerging trends outside of the research scope and available historical data sets may be ignored. This inference is based on statistics where the objective function (known as dependent variable) calculated based on a series of variables which are assumed to be independent. Although, simplification has been a traditional useful solution paradigm to interpret a system and develop a model to discuss how it works when complexity rises the reliability falls. Unlike classic statistical prediction models which aimed to extrapolate future behaviors of the objective variable based on a series of independent variables, disciplines such as data mining brought a solution. Data mining ignored the mentioned simplifying assumption and instead it assumes in the real world variables are correlated but with varying degrees (Hafezi, Shahrabi, and Hadavandi 2015). Data mining (DM) is defined as the process of extracting appealing patterns and deriving knowledge in massive datasets (Hafezi, Akhavan, Zamani, et al. 2019). Refer to Han et al.: "the principal dimensions are data, knowledge, applications, and technologies" (Han, Pei, and Kamber 2011). Moreover, machine learning is used extensively to predict in the case of complex systems and dynamic environments (Hafezi and Akhavan 2018, Lotfinejad et al. 2018). Alpaydin defined machine learning (ML) as "programming computers to optimize a performance criterion using example data or past experience" (Alpaydin 2009). ML can be challenging for many real-world problems for which creating a detailed design can be quite hard despite a clear specification (Rebala, Ravi, and Churiwala 2019). Although ML deals with historical time series it is not limited to a database problem; it is also a part of artificial intelligence (AI) (Alpaydin 2009). To be intelligent, a technique should have the ability to learn. Through ML we will have a model of some variables and parameters, developed based on a learning process via a computer program aimed to optimize parameters using past experiences, known as training data. Application of ML to large data bases is called DM (Kavakiotis et al. 2017). Generally, the model can be (1) predictive in order to provide predictions about future, (2) descriptive which fundamentally concerns with extracting knowledge through a dataset or (3) both (Jain and Srivastava 2013, Hand 2006).

In the 1980s, approaches to crafting rules to solve complex problems were quite unsuccessful (Rebala, Ravi, and Churiwala 2019). Moreover, there are some impractical conventional solutions which are applied individually and cannot be generalized. In such a condition, a learning model is needed. When human expertise does not exist or even in a condition that experts are unable to explain their experiences learning models are essential. Sometimes different people explain the same experience in different manners as they have different interprets, ages, gender or even accent. Moreover, in cases the problem changes in time or actions can change conditions. ML-based models can overcome in such a dynamic environment.

Technological growth in recent years has improved computational capabilities dramatically and as result learning models are available and applicable. But what are learning models' contributions to futures studies? It is a challenging question which this paper attempts to address via noting some successful experiences. As FS has strong relationships with policy- 
making and strategic management disciplines qualitative methodologies shaped most previous attempts in the field of FS and foresight. However, as many previous studies noted, a mixes qualitative-quantitative methodology leads to a better understanding (Amer, Daim, and Jetter 2013, Hafezi, Akhavan, Pakseresht, et al. 2019, Alipour et al. 2017). To represents how ML, $\mathrm{AI}$, and DM can contribute to a futures study as a predictor some previous prediction applications in a complex environment are reviewed (the following section) then the usefulness of a DM will be studied to extract driving forces.

\section{Review on using artificial intelligence to predict future trends:}

Although many notable researchers have used artificial intelligence to perform predictions in dynamic and complex systems (Hafezi, Shahrabi, and Hadavandi 2015) it has been rarely used by futurists as a tool to recognize patterns and extrapolate trends. Delic claimed that intelligent systems will play a significant role in a mid-term future (Delic 2006) (see figure 2).

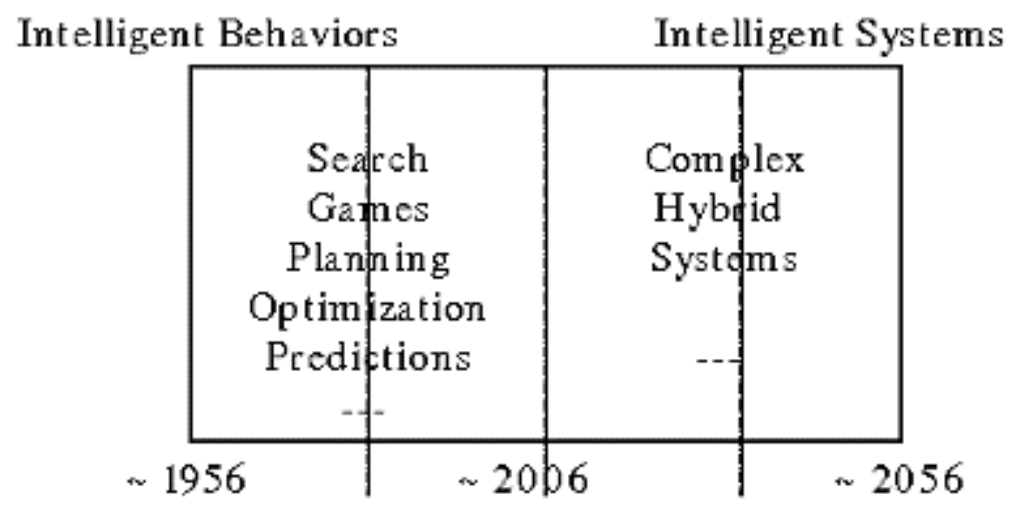

Figure 2. Long view of AI research (Delic 2006)

Time series (for instance (Sen, Roy, and Pal 2016, Fagiani et al. 2015, Shi, Liu, and Wei 2016, Zhang and Yang 2015, Taşpınar, Celebi, and Tutkun 2013, Ervural, Beyca, and Zaim 2016, Zhu et al. 2015)), regression (for instance (Dalfard et al. 2013, Bianco, Scarpa, and Tagliafico 2014, Baldacci et al. 2016)) and econometrics (for instance (Adams and Shachmurove 2008, Ramanathan 2006, Iniyan, Suganthi, and Samuel 2006)) were used widely to study future behaviors, which generally are developed for stock market predictions. Among all benefits traditional models have some crucial challenges, (1) focusing on historical data with a fundamental assumption which claims the future follows the past, (2) jumps and drips are not considered in the model and (3) they do not use root tests and feedbacks to estimate parameters. Instead, though similarly, learning models utilize historical time series generally these models are able to cope with fluctuations and use feedbacks to upgrade model adjustment parameters. Artificial neural networks (van Gerven and Bohte 2018, Wasserman 1993, Zurada 1992), genetic algorithm (Koza 1997, Davis 1991, Kramer 2017), support vector machines (Meyer and Wien 2015, Guenther and Schonlau 2016), particle swarm optimization (Shi 2001, Kennedy 2010, Marini and Walczak 2015) and fuzzy inference systems (Lin et al. 1996, Jang 1993) are some well-known and used learning models that have been applied in different contexts. 
Among all successful and valuable studies, Hafezi et al. used a learning-based prediction model to project DAX stock prices in the period of 2000's financial crisis (Hafezi, Shahrabi, and Hadavandi 2015). Results showed that the developed learning model which is developed based on artificial neural networks overcame benchmark prediction models. Moreover, in the case of energy systems, which are also known as complex systems (Hafezi, Akhavan, and Pakseresht 2017), Aydinalp-Koksal and Ugursal (Aydinalp-Koksal and Ugursal 2008) modeled residential end-use-energy demand using a conditional demand analysis (CDA) approach. The prediction performance of the CDA model was compared with an earlier artificial neural network and a computational model. The results obtained by the CDA were shown to be superior to the alternative models they considered. Shimoda et al. (Shimoda et al. 2010) presented a simulation model to study the energy demand of the residential sector at the national level, studying the variety of different households and building types. Roger (Rodger 2014) addressed specifically natural gas demand prediction issues. His system was based on an artificial neural network structure to create a fuzzy-nearest-neighbor neural network model. As it is obvious learning models showed a great performance to predict in dynamic and chaotic environments. However, a single prediction rose the risk level and moreover, FS is known as open to the future. From a technical perspective, a single deterministic prediction approach cannot adequately support the decision-making process for the complex and uncertain natural gas markets. In simple words, FS aimed to develop alternative projections which define directions of scenarios.

\section{Examples of how learning models can equip FS:}

Scenario development is known as a well-used FS methodology (Hafezi and Akhavan 2019, Alipour et al. 2018). Scenarios are used to produce forecasts of future business environments and to identify conditions leading to major changes in these environments (Huss and Honton 1987). Generally, scenarios contain stories about possible futures (Bishop, Hines, and Collins 2007). Moreover, scenario planning attempts to address uncertainties and facilitate planning through clarifying future milieu (Goodwin and Wright 2001, Schoemaker 1995). Some previous studies attempted to develop a scenario methodology based on quantitative methods such as simulation (Rausch and Catanzaro 2003, Duperrin and Godet 1975, Hughes 1999), probability trees (Montreuil and Laforge 1992) and fuzzy cognitive maps (Amer, Jetter, and Daim 2011, Kim et al. 2016, Alipour et al. 2017). Recent years learning models are used, though limited, to develop scenarios and purify driving forces. As an example, a recent study is investigated. Hafezi et al. (Hafezi, Akhavan, Zamani, et al. 2019) tested a hypothesis to prove the usefulness of learning models to purify driving forces in a complex system. Driving forces are those features which shape the future and a decision-maker can interpret upcoming trends refer to driving forces different orientations. Driving forces must be relatively independent, but when facing a large dataset it is not simple to analyze and evaluate whether different features are independent or correlated. DM help researchers to select the best subset among all existing input features. A subset with fewer features means a reduction in problem dimensions. Consequently, DM will cause saving time and cost, simultaneously improving interpretability. Moreover, Hafezi et al. have shown using DM can improve the reliability in the term of satisfying multi error criteria and accuracy as it overcame the same estimator but with no data mining based preprocessing step. Table 1 summarizes the difference between an equipped and unequipped model refer to four various error statistics; R $^{2}$, MAE, MAPE and RMSE. 
Table 1. Statistical errors for different input vectors (raw versus processed) based on (Hafezi, Akhavan, Zamani, et al. 2019)

\begin{tabular}{ccccc}
\hline Input protocol & $\mathbf{R}^{2}$ & MAE & MAPE & RMSE \\
\hline Processed data & $\underline{\mathbf{0 . 9 8 4 7}}$ & $\underline{\mathbf{5 2 . 1 9}}$ & $\underline{\mathbf{1 . 6 9}}$ & $\underline{\mathbf{6 1 . 3 3}}$ \\
Raw data & 0.9679 & 79.96 & 2.61 & 94.21 \\
\hline
\end{tabular}

In this research authors extracted a subset of 13 features contains only 6 features (the input vector dimension reduced almost about 50\%) using a feature selection method named correlation-based feature selection (CFS) (proposed by hall (Hall 1999)). DM based feature selection guarantees to reduce problem space (i.e. complexity) while ensures no informative input will be neglected.

In addition, to showing how $\mathrm{AI}$ and DM can contribute to the FS, a Ph.D. dissertation done in the field of technology foresight at Tehran Polytechnic (Hafezi 2018) which developed a scenario development model based on artificial intelligence, Monte Carlo simulation and data mining preprocessing to generate potential scenarios for Iran's natural gas export futures. In this case, similar to the research mentioned above, DM techniques were implemented to extract the most important features which shape the future and can help to evaluate future trends. Then AI was used to generate the reference case. The reference case scenario was compared to other published works to evaluate if it follows business as usual scenario proposed by energy agencies or not. Finally, refer to selected feature subset, different global policies were evaluated and different scenarios were developed to project future global natural gas demand and their deviation from the reference case. Now policymakers are equipped with a scenario model which can project consequences of various policies. Moreover, sensitivity analysis is applicable to evaluate features refer to the size of effects on outputs.

\section{Discussions and conclusions:}

The world approaching the industry 4.0 era where human-machine interaction going to change markets, industries and even living paradigms and quality (Gorecky et al. 2014, Hofmann and Rüsch 2017, Lom, Pribyl, and Svitek 2016). In today's complex modern world, FS is going to address new challenges and as a result, it needs more flexible and powerful tools to recognize pattern behind chaotic raw data. Availability of datasets, updated computing technologies, and improved learning algorithms will facilitate the convergence of long-term strategic thinking and data science. Numerous studies investigated applications of expert systems and artificial intelligence to support decision-makers, however still this emerging field suffers from lack of compatible methodologies and even the resistance of the scientific community, especially among futurists. Now most FS reports, even in the academic atmosphere, is based on exportoriented qualitative methodologies. Elitism is criticized (see (Bachrach 1967, Anderson 1988, Sartori 1978)) while FS fundamentally aimed to facilitate democracy and rational policymaking process. Using data facilitates a comprehensive review as data is rarely biased to specific thinking paradigm. 
As an interdisciplinary field, FS needs to equip with data mining, pattern recognition, machine learning and etc. to obtain more reliable outcomes/ decision supports. In this paper, some successful researches in the case of complex systems' prediction and scenario development were reviewed which used AI as their central core. Findings showed that though little effort has been made successful applications support developments.

\section{References:}

Adams, F Gerard, and Yochanan Shachmurove. 2008. "Modeling and forecasting energy consumption in China: Implications for Chinese energy demand and imports in 2020." Energy economics 30 (3):1263-1278.

Alipour, M, R Hafezi, Muhammad Amer, and AN Akhavan. 2017. "A new hybrid fuzzy cognitive map-based scenario planning approach for Iran's oil production pathways in the post-sanction period." Energy 135:851-864.

Alipour, Mohammad, Reza Hafezi, Bilal Ervural, Mohamad Amin Kaviani, and Özgür Kabak. 2018. "Long-term policy evaluation: Application of a new robust decision framework for Iran's energy exports security." Energy 157:914-931.

Alpaydin, Ethem. 2009. Introduction to machine learning: MIT press.

Amer, Muhammad, Tugrul U Daim, and Antonie Jetter. 2013. "A review of scenario planning." Futures 46:23-40.

Amer, Muhammad, Antonie Jetter, and Tugrul Daim. 2011. "Development of fuzzy cognitive map (FCM)-based scenarios for wind energy." International Journal of Energy Sector Management 5 (4):564-584.

Anderson, Talmadge. 1988. "Black encounter of racism and elitism in white academe: A critique of the system." Journal of Black Studies 18 (3):259-272.

Aydinalp-Koksal, Merih, and V Ismet Ugursal. 2008. "Comparison of neural network, conditional demand analysis, and engineering approaches for modeling end-use energy consumption in the residential sector." Applied Energy 85 (4):271-296.

Bachrach, Peter. 1967. The theory of democratic elitism: A critique: Little, Brown Boston.

Baldacci, Lorenzo, Matteo Golfarelli, Davide Lombardi, and Franco Sami. 2016. "Natural gas consumption forecasting for anomaly detection." Expert Systems with Applications 62:190-201.

Bianco, Vincenzo, Federico Scarpa, and Luca A Tagliafico. 2014. "Analysis and future outlook of natural gas consumption in the Italian residential sector." Energy Conversion and Management 87:754-764.

Bishop, Peter, Andy Hines, and Terry Collins. 2007. "The current state of scenario development: an overview of techniques." foresight 9 (1):5-25.

Dalfard, V Majazi, M Nazari Asli, SM Asadzadeh, SM Sajjadi, and A Nazari-Shirkouhi. 2013. "A mathematical modeling for incorporating energy price hikes into total natural gas consumption forecasting." Applied Mathematical Modelling 37 (8):5664-5679.

Davis, Lawrence. 1991. "Handbook of genetic algorithms."

de Miranda Santo, Marcio, Gilda Massari Coelho, Dalci Maria dos Santos, and Lélio Fellows Filho. 2006. "Text mining as a valuable tool in foresight exercises: A study on nanotechnology." Technological Forecasting and Social Change 73 (8):1013-1027. 
Delic, Kemal A. 2006. "AI re-emerging as research in complex systems." Ubiquity 2006 (October):1.

Duperrin, JC, and Michel Godet. 1975. "SMIC 74-a method for constructing and ranking scenarios." Futures 7 (4):302-312.

Ervural, Beyzanur Cayir, Omer Faruk Beyca, and Selim Zaim. 2016. "Model Estimation of ARMA Using Genetic Algorithms: A Case Study of Forecasting Natural Gas Consumption." Procedia-Social and Behavioral Sciences 235:537-545.

Fagiani, Marco, Stefano Squartini, Leonardo Gabrielli, Susanna Spinsante, and Francesco Piazza. 2015. "A review of datasets and load forecasting techniques for smart natural gas and water grids: Analysis and experiments." Neurocomputing 170:448-465.

Goodwin, Paul, and George Wright. 2001. "Enhancing strategy evaluation in scenario planning: a role for decision analysis." Journal of management studies 38 (1):1-16.

Gorecky, Dominic, Mathias Schmitt, Matthias Loskyll, and Detlef Zühlke. 2014. "Humanmachine-interaction in the industry 4.0 era." 2014 12th IEEE international conference on industrial informatics (INDIN).

Guenther, Nick, and Matthias Schonlau. 2016. "Support vector machines." The Stata Journal 16 (4):917-937.

Hafezi, R. 2018. "Designing a conceptual scenario model to project Iran's natural gas export futures " PhD, Management, Science \& Technology, Amirkabir University of Technology (Tehran Polytechnic).

Hafezi, Reza, and Amir Akhavan. 2018. "Forecasting Gold Price Changes: Application of an Equipped Artificial Neural Network." AUT Journal of Modeling and Simulation 50 (1):71-82.

Hafezi, Reza, and Amir Naser Akhavan. 2019. "A Novel Conceptual Risk Management Model Based on The Future's Uncertainties."

Hafezi, Reza, Amir Naser Akhavan, Mazdak Zamani, Saeed Pakseresht, and Shahab Shamshirband. 2019. "Developing a Data Mining Based Model to Extract Predictor Factors in Energy Systems: Application of Global Natural Gas Demand."

Hafezi, Reza, AmirNaser Akhavan, and Saeed Pakseresht. 2017. "Projecting plausible futures for Iranian oil and gas industries: Analyzing of historical strategies." Journal of Natural Gas Science and Engineering 39:15-27.

Hafezi, Reza, AmirNaser Akhavan, Saeed Pakseresht, and David A Wood. 2019. "A Layered Uncertainties Scenario Synthesizing (LUSS) model applied to evaluate multiple potential long-run outcomes for Iran's natural gas exports." Energy 169:646-659.

Hafezi, Reza, Siavosh Malekifar, and Amirnaser Akhavan. 2018. "Analyzing Iran's science and technology foresight programs: recommendations for further practices." foresight 20 (3):312-331.

Hafezi, Reza, Jamal Shahrabi, and Esmaeil Hadavandi. 2015. "A bat-neural network multiagent system (BNNMAS) for stock price prediction: Case study of DAX stock price." Applied Soft Computing 29:196-210.

Hall, Mark Andrew. 1999. "Correlation-based feature selection for machine learning."

Han, Jiawei, Jian Pei, and Micheline Kamber. 2011. Data mining: concepts and techniques: Elsevier.

Hand, David J. 2006. "Data Mining." Encyclopedia of Environmetrics 2. 
Hofmann, Erik, and Marco Rüsch. 2017. "Industry 4.0 and the current status as well as future prospects on logistics." Computers in Industry 89:23-34.

Hughes, Barry B. 1999. "The international futures (IFs) modeling project." Simulation \& Gaming 30 (3):304-326.

Huss, William R, and Edward J Honton. 1987. "Scenario planning-what style should you use?" Long range planning 20 (4):21-29.

Ian Miles, CRIC. 2002. "Appraisal of alternative methods and procedures for producing Regional Foresight."

Iniyan, Suganthi, L Suganthi, and Anand A Samuel. 2006. "Energy models for commercial energy prediction and substitution of renewable energy sources." Energy Policy 34 (17):2640-2653.

Jain, Nikita, and Vishal Srivastava. 2013. "Data mining techniques: a survey paper." IJRET: International Journal of Research in Engineering and Technology 2 (11):2319-1163.

Jang, J-SR. 1993. "ANFIS: adaptive-network-based fuzzy inference system." IEEE transactions on systems, man, and cybernetics 23 (3):665-685.

Kavakiotis, Ioannis, Olga Tsave, Athanasios Salifoglou, Nicos Maglaveras, Ioannis Vlahavas, and Ioanna Chouvarda. 2017. "Machine learning and data mining methods in diabetes research." Computational and structural biotechnology journal 15:104-116.

Keenan, Michael, and R Popper. 2007. "Combining foresight methods for impacts." NISTEP 3rd International Conference on Foresight. Tokio.

Kennedy, James. 2010. "Particle swarm optimization." Encyclopedia of machine learning:760-766.

Kim, Jieun, Mintak Han, Youngjo Lee, and Yongtae Park. 2016. "Futuristic data-driven scenario building: Incorporating text mining and fuzzy association rule mining into fuzzy cognitive map." Expert Systems with Applications 57:311-323.

Koza, John R. 1997. "Genetic programming."

Kramer, Oliver. 2017. Genetic algorithm essentials. Vol. 679: Springer.

Lin, Chin-Teng, CS George Lee, Chin-Teng Lin, and CT Lin. 1996. Neural fuzzy systems: a neuro-fuzzy synergism to intelligent systems. Vol. 205: Prentice hall PTR Upper Saddle River NJ.

Lom, Michal, Ondrej Pribyl, and Miroslav Svitek. 2016. "Industry 4.0 as a part of smart cities." 2016 Smart Cities Symposium Prague (SCSP).

Lotfinejad, Mohammad, Reza Hafezi, Majid Khanali, Seyed Hosseini, Mehdi Mehrpooya, and Shahaboddin Shamshirband. 2018. "A comparative assessment of predicting daily solar radiation using bat neural network $(\mathrm{BNN})$, generalized regression neural network (GRNN), and neuro-fuzzy (NF) system: A case study." Energies 11 (5):1188.

Marini, Federico, and Beata Walczak. 2015. "Particle swarm optimization (PSO). A tutorial." Chemometrics and Intelligent Laboratory Systems 149:153-165.

Martin, Ben R, and Ron Johnston. 1999. "Technology foresight for wiring up the national innovation system: experiences in Britain, Australia, and New Zealand." Technological forecasting and social change 60 (1):37-54.

Meissner, Dirk. 2012. "Results and impact of national Foresight-studies." Futures 44 (10):905913. 
Meyer, David, and FH Technikum Wien. 2015. "Support vector machines." The Interface to libsvm in package e 1071:28.

Montreuil, Benoit, and Andrée Laforge. 1992. "Dynamic layout design given a scenario tree of probable futures." European Journal of Operational Research 63 (2):271-286.

Pillkahn, Ulf. 2008. Using trends and scenarios as tools for strategy development: shaping the future of your enterprise: John Wiley \& Sons.

Popper, Rafael. 2008a. "Foresight methodology." The handbook of technology foresight:4488.

Popper, Rafael. 2008b. "How are foresight methods selected?" foresight 10 (6):62-89.

Prior, Tim, Jane Daly, Leah Mason, and Damien Giurco. 2013. "Resourcing the future: Using foresight in resource governance." Geoforum 44:316-328.

Ramanathan, Ramakrishnan. 2006. "A multi-factor efficiency perspective to the relationships among world GDP, energy consumption and carbon dioxide emissions." Technological Forecasting and Social Change 73 (5):483-494.

Rausch, Erwin, and Frank Catanzaro. 2003. "Simulation and games in futuring and other uses." Futures Research Methodology (Version 2.0), AC/UNU Millennium Project, Washington, DC.

Rebala, Gopinath, Ajay Ravi, and Sanjay Churiwala. 2019. "Machine Learning Definition and Basics." In An Introduction to Machine Learning, 1-17. Springer.

Rodger, James A. 2014. "A fuzzy nearest neighbor neural network statistical model for predicting demand for natural gas and energy cost savings in public buildings." Expert Systems with Applications 41 (4):1813-1829.

Sartori, Giovanni. 1978. "Anti-elitism revisited." Government and Opposition 13 (1):58-80.

Schoemaker, Paul JH. 1995. "Scenario planning: a tool for strategic thinking." Sloan management review 36 (2):25-50.

Sen, Parag, Mousumi Roy, and Parimal Pal. 2016. "Application of ARIMA for forecasting energy consumption and GHG emission: A case study of an Indian pig iron manufacturing organization." Energy 116:1031-1038.

Shi, Guang, Derong Liu, and Qinglai Wei. 2016. "Energy consumption prediction of office buildings based on echo state networks." Neurocomputing 216:478-488.

Shi, Yuhui. 2001. "Particle swarm optimization: developments, applications and resources." Proceedings of the 2001 congress on evolutionary computation (IEEE Cat. No. 01TH8546).

Shimoda, Yoshiyuki, Yukio Yamaguchi, Tomo Okamura, Ayako Taniguchi, and Yohei Yamaguchi. 2010. "Prediction of greenhouse gas reduction potential in Japanese residential sector by residential energy end-use model." Applied Energy 87 (6):19441952.

Smith, Jack E, and Ozcan Saritas. 2011. "Science and technology foresight baker's dozen: a pocket primer of comparative and combined foresight methods." Foresight 13 (2):7996.

Taşpınar, Fatih, Numan Celebi, and Nedim Tutkun. 2013. "Forecasting of daily natural gas consumption on regional basis in Turkey using various computational methods." Energy and Buildings 56:23-31. 
van Gerven, Marcel, and Sander Bohte. 2018. Artificial neural networks as models of neural information processing: Frontiers Media SA.

Vecchiato, Riccardo, and Claudio Roveda. 2014. "Foresight for public procurement and regional innovation policy: The case of Lombardy." Research Policy 43 (2):438-450.

Vishnevskiy, Konstantin O, and Olga G Egorova. 2015. "Strategic foresight for SMEs: choice of the most relevant methods." ISPIM Conference Proceedings.

Wasserman, Philip D. 1993. Advanced methods in neural computing: John Wiley \& Sons, Inc.

Zhang, Wei, and Jun Yang. 2015. "Forecasting natural gas consumption in China by Bayesian model averaging." Energy Reports 1:216-220.

Zhu, L, MS Li, QH Wu, and L Jiang. 2015. "Short-term natural gas demand prediction based on support vector regression with false neighbours filtered." Energy 80:428-436.

Zurada, Jacek M. 1992. Introduction to artificial neural systems. Vol. 8: West publishing company St. Paul.

Zweck, A. 2002. "Three perspectives for one future in economy and society." Futures Research Quarterly 18 (1):55-66. 